\title{
SEKONDER GÖZ İÇİ LENS İMPLANTASYONU NEDENLERİ VE CERRAHI SONUÇLARIMIZ
}

\author{
Causes of Secondary Intraocular Lens Implantation and Surgical Outcomes
}
Tevfik OĞUREL ${ }^{1}$, Nesrin BÜYÜKTORTOP GÖKÇINAR ${ }^{2}$, Yaprak AKBULUT ${ }^{3}$, Fatma ÖZKAL $^{4}$, Nurgül ÖRNEK ${ }^{5}$, Zafer ONARAN ${ }^{6}$

1,2,3,4,5,6 Kırıkkale Üniversitesi Tıp Fakültesi, Göz Hastalıkları Anabilim Dalı, KIRIKKALE, TÜRKIYE

Amaç: Sekonder göz içi lens (GIL) implantasyonu yapılan hastalarda, preoperatif oküler risk faktörlerini ve ikincil GíL cerrahisi endikasyonlarını belirlemek ve bu işlemin görsel sonuçlarını karşılaştırmak amacıyla yapılacaktır.

Gereç ve Yöntemler: Kırıkkale Üniversitesi Tıp Fakültesi'nde, Ocak 2018 ve Şubat 2019 tarihleri arasında sekonder GIL implantasyonu yapılan ve postoperatif en az 3 ay takibi olan 19 hasta çalışmaya dâhil edildi. Ameliyat öncesinde hastaların sekonder GIL implantasyonu nedenleri; psödoeksfoliyasyon (PEKS), üveit gibi ek oküler patolojiler, travma, sistemik sendromlar, ameliyat öncesi ve sonrası düzeltilmiş en iyi görme keskinlikleri (DEGK), uygulanan cerrahi yöntem ve olası postoperatif komplikasyonlar ayrı ayrı not edildi.

Bulgular: Çalışmaya 19 hastanın 19 gözü dahil edildi. Hastaların 11 (\%57.9)'i kadın, 8 (\%42.1)'i erkekti. Tüm hastaların yaş ortalaması $69.16 \pm 6.92 \quad(59-80)$ idi. Hastalar operasyon öncesi değerlendirildiğinde, en s1k neden, primer cerrahi sonrası intraoperatif komplikasyonlara sekonder gelişen cerrahi afaki idi (\%52.6, $\mathrm{n}=11)$. İkinci en s1k neden ise 6 hastada görülen GiL subluksasyonu idi. Sulkus desteği olan hastalara sulkus yerleşimli GILL diğer hastalara ise, iris ya da skleral fiksasyonlu GIL implantasyonu yapıldı. Hastaların cerrahi öncesi DEGK $0.223 \pm 0.187$ iken cerrahi sonras1 DEGK $0.702 \pm 0.209$ idi. Ameliyat öncesi ve sonrası görme düzeyleri değerlendirildiğinde aradaki fark istatistiksel olarak ileri düzeyde anlamlıydı $(\mathrm{p}<0.001)$.

Sonuç: Sulkus yerleşimli GİL implantasyonu, iris ve skleral fiksasyonlu GİL implantasyonu yöntemlerine göre daha kolay ve komplikasyon oranı daha düşük bir yöntemdir. Sulkus desteğinin olmadığı durumlarda iris fiksasyonu ya da skleral fiksasyon yönteminin sonuçları da yüz güldürücüdür.

Anahtar Kelimeler: Sekonder GIL implantasyonu, katarakt, GÍL subluksasyonu
Objective: The aim of this study is to determine preoperative ocular risk factors and indications for secondary intraocular lens (IOL) implantation surgery and to compare the visual outcomes of this procedure.

Material and Methods: Nineteen patients who underwent secondary IOL implantation between January 2018 and February 2019 at Kırıkkale University Faculty of Medicine were included in the study. Preoperative causes of secondary IOL implantation, additional ocular pathologies such as pseudoexfoliation (PEX), uveitis, trauma, systemic syndromes, best corrected visual acuity (BCVA) before and after surgery, surgical procedures and possible postoperative complications were noted separately.

Results: Nineteen eyes of 19 patients were included in this study. Eleven $(57.9 \%)$ of the patients were female and 8 $(42.1 \%)$ were male. The mean age of all patients was $69.16 \pm$ $6.92(59-80)$ years. When the patients were evaluated preoperatively, the most common cause was surgical aphakia secondary to intraoperative complications due to primary surgery in 11 patients $(52.6 \%)$. The second most common cause was IOL subluxation in 6 patients. IOL implantation with iris or scleral fixation was performed in patients who did not have sulcus support and the other IOLs were inserted to the sulcus. Preoperative mean BCVA was $0.223 \pm 0.187$, and postoperative mean BCVA was $0.702 \pm 0.209$. The difference between preoperative and postoperative mean BCVA was statistically significant $(\mathrm{p}<0.001)$.

Conclusion: IOL implantation in the sulcus is easier and the complication rate is lower than the iris and scleral fixation IOL implantation methods. The results of iris fixation or scleral fixation in cases without sulcus support are also promising.

Keywords: Secondary IOL implantation, cataract, IOL subluxation 


\section{GİRIȘ}

İdeal olarak, sorunsuz katarakt ameliyatından sonra, göz içi lensi (GİL) kapsüler kese içine implante edilir. Fakat GİL subluksasyonu, gevşek zonül gibi kapsüler kese ile ilgili preoperatif problemler yâda kapsülde olabilecek intraoperatif komplikasyonlar nedeniyle bu her zaman mümkün olmayabilir. Bu durumda GİL; iris, sulkus yâda sklera gibi farklı pozisyonlarda fikse edilebilir. İntraoperatif komlikasyonlarda bu yöntemler, cerrahın tecrübesi ve cerrahinin süresine bağlı olarak aynı seansta da yapılabilirken, genellikle ve GİL subluksasyonu, yanlış GİL, GİL opaklaşması, üveit glokom hifema (UGH) sendromu ya da sekonder afaki gibi nedenlerden dolayı ikinci bir seansta GÍL yerleştirilmektedir.

$\mathrm{Bu}$ retrospektif çalışma, preoperatif oküler risk faktörlerini ve ikincil GİL cerrahisi endikasyonlarını belirlemek ve bu işlemin görsel sonuçlarını karşılaştırmak amacıyla yapılacaktır. İkincil bir analiz, preoperatif oküler risk faktörleri, uygulanan cerrahi yöntemleri ve postoperatif komplikasyonların refraktif ve görsel sonuç üzerindeki etkisini inceleyecektir.

\section{MATERYAL VE METOD}

Kırıkkale Üniversitesi Tıp Fakültesi'nde, Ocak 2018 ve Şubat 2019 tarihleri arasında sekonder GİL implantasyonu yapılan ve postoperatif en az 3 ay takibi olan 19 hasta çalışmaya dâhil edildi. Hastaların dosyaları retrospektif olarak incelendi. Her vaka ayrı ayrı değerlendirildi. Ameliyat öncesi hastaların yaş, cinsiyet, ameliyat tarihi, primer katarakt ameliyatı tarihi, sekonder GİL implantasyonu endikasyonu; psödoeksfoliyasyon (PEKS), üveit gibi ek oküler patolojiler, travma, sistemik sendromlar, ameliyat öncesi ve sonrası düzeltilmiş en iyi görme keskinlikleri (DEGK), uygulanan cerrahi yöntem ve olas1 postoperatif komplikasyonlar ayrı ayrı not edildi.

\section{Istatistiksel Analiz}

İstatistiksel değerlendirme için SPSS 20.0 programı kullanıldı. Sayısal değerler gösteren verilerin normal dağılım gösterip göstermediği Kolmogorov-Smirnov testi ile değerlendirildi. Normal dağılım göstermeyen veriler için Mann-Whitney $U$ testi, kategorik değişkenler için Pearson'ın kikare testi kullanıldı. $\mathrm{p}<0.05$ istatistiksel olarak anlamlı kabul edildi.

\section{BULGULAR}

Çalışmaya 19 hastanın 19 gözü dahi edildi. Hastaların 11 (\%57.9)'i kadın, 8 (\%42.1)'i erkekti. Tüm hastaların yaş ortalaması, $69.16 \pm 6.92(59-80)$ yı1 idi. Hastaların demografik özellikleri Tablo 1'de gösterilmiştir. Hastalar operasyon öncesi değerlendirildiğinde en s1k neden, 11 hastada (\%52.6) primer cerrahiye sonras1, intraoperatif komplikasyonlara sekonder gelişen cerrahi afaki idi. İkinci en sık neden ise 6 hasta da görülen GİL subluksasyonu idi. Bunlardan 5 hastada psodoeksfolyasyon (PEKS)'a bağlı zonüler defekt mevcutken, bir hastada ise, primer cerrahide yerleştirilen GİL'in haptiğinin kopması idi. Sekonder GIL iplantasyonu yapılan hastaların etiyolojik nedenleri Tablo 2'de gösterilmiştir.

Tablo 1: Sekonder GİL implantasyonu yapilan hastaların demografik özellikleri (GİL: Göz İçi Lensi)

\begin{tabular}{lc}
\hline Parametre & Ortalama \\
\hline Yaş (yıl) & $69.16 \pm 6.92$ \\
Takip süresi (ay) & $5.47 \pm 2.24$ \\
Cinsiyet & $\mathrm{n}(\%)$ \\
\hline Erkek & $8(42.1)$ \\
Kadın & $11(57.9)$ \\
\hline
\end{tabular}

Afak olan hastaların dördünde GİL sulkusa yerleştirilirken, 3 hastaya iris fiksasyonlu, 4 hastayada skleral fiksasyonlu GİL implantasyonu yapıldı. GİL subluksayonu olan hastalarda tercih edilen yöntem, göz içinde üst haptiğin bag ile birlikte sütüre edilerek skleraya fikse edilmesi şeklindeydi. Haptik defekti nedeniyle sublukse olan hastada ise, GİL göz içinde 
kesilerek çıkartıldı ve sekonder GíL bag içine yerleştirildi. Yanlış GİL gücü hesaplanan ve GİL opasifikasyonu olan hastalarada da aynı yöntem uyguland1.

Hastaların cerrahi öncesi DEGK $0.223 \pm 0.187$ iken, cerrahi sonrası DEGK 0.702 \pm 0.209 idi. Ameliyat öncesi ve sonrası görme düzeyleri değerlendirildiğinde aradaki fark istatistiksel olarak ileri düzeyde anlamlıydı $(\mathrm{p}<0.001)$.

İris fiksasyonu yapılan bir hastada UGH sendromu gelişmesi nedeniyle, başka bir seansta, GİL skleraya fikse edilirken, skleral fiksasyon yapılan bir hastada tek bacakta gelişen sütür kopması nedeniyle GİL tekrar repoze edilerek sütüre edildi. Skleral fiksasyon yapılan başka bir hastada sütürlerin-haptiklerin aşırı gergin olması nedeniyle görülen yüksek astigmatizma mevcuttu. Hasta reoprasyonu kabul etmediğinden herhangi bir müdahale yapılmadı. Uygulanan cerrahi yönteme göre postoperatif ortalama DEGK ve görülen komplikasyonlar Tablo 3’te gösterilmiştir.

Tablo 2: Sekonder GİL implantasyonu yapılan hastalarda etyolojik nedenler ve uygulanan cerrahi yöntem (PEKS: Psodoeksfolyasyon, GİL: Göz içi lensi)

\begin{tabular}{lcccc}
\hline Etyoloji & \multicolumn{3}{l}{ Uygulanan cerrahi yöntem } \\
\hline \multirow{2}{*}{ Cerrahi Afaki } & Kapsüler Kese & Sulkus & Skleral Fiksasyon & Iris Fiksasyon \\
\hline GÍL Subluksasyonu & & 4 & 3 \\
\hline PEKS & 1 & & \\
\hline Haptik Kopması & 1 & & \\
\hline Yanlıs GIL Gücü & 1 & & \\
\hline GIL Opasifikasyonu & & & \\
\hline
\end{tabular}

Tablo 3: Uygulanan cerrahi yönteme göre postoperatif ortalama görme düzeyi ve görülen komplikasyonlar (DEGK: Düzeltilmiş en iyi görme keskinliği)

\begin{tabular}{|c|c|c|c|c|}
\hline \multirow[b]{3}{*}{ DEGK } & \multicolumn{4}{|c|}{ Uygulanan cerrahi yöntem } \\
\hline & Kapsüler Kese & Sulkus & Skleral Fiksasyon & İris Fiksasyon \\
\hline & $0.96 \pm 0.05$ & $0.90 \pm 0.11$ & $0.46 \pm 0.23$ & $0.90 \pm 0.17$ \\
\hline \multicolumn{5}{|l|}{ Komplikasyon } \\
\hline UGH sendromu & & & & 1 \\
\hline Sütür Kopması & & & 1 & \\
\hline Gergin Sütür & & & 1 & \\
\hline
\end{tabular}

\section{TARTIŞMA}

Sekonder GİL, GİL değişimi sırasında veya afakiyi tedavi etmek için implante edilir (1). GİL değişimi için en yaygın endikasyonlar GİL dislokasyonu, UGH sendromu, hatalı GİL gücü, GİL opaklaşması ve multifokal GİL'den memnuniyetsizliktir (2-5).
Çalışmamızda da herhangi bir nedenden dolayı gelişen afaki ve GİL dislokasyonu, sekonder GİL implantasyonunun major nedenlerini oluşturmaktaydı. Sekonder GİL’nin kapsüler keseye yerleştirilememesi durumunda, alternatif farklı seçenekler bulunmaktadır. Kapsül desteğinin devam ettiği olgularda en ideal 
seçenek, 3 parçalı bir GİL'in siliyer sulkusa yerleştirilmesidir (6). GİL'nin sulkusa yerleştirilmesi diğer yöntemlere göre hem daha basit hem de tolere edilebilen bir yöntemdir. Çünkü GİL, bir sütürasyon ya da fiksasyona gerek kalmadan sulkus desteği üzerinde kalabilir. Nitekim çalışmamızda da sulkus desteği olan hastalarda postoperatif komplikasyon oranı daha düşük ve iyileşme süreci daha hızlı idi. Sulkusa yerleştirilen GİL'in dezavantajı, potansiyel olarak UGH sendromu ile sonuçlanabilen GİL haptiğinin irise sürtünme riskidir (7). Çalışmamızda sulkusa yerleştirlen hiçbir hastada böyle bir komplikasyonla karşılaşılmadı. Özellikle travma ya da komplike cerrahiler nedeniyle kapsül desteğinin olmadığı durumlarda, ön kamera, iris fiksasyon ya da skleral fiksasyon gibi alternatif yöntemler mevcuttur (8). Her tekniğin kendine özgü yararları ve zorlukları vardır ve verilecek olan karar eşlik eden oküler patoloji ve cerrahın tecrübesine göre olur (9).

Ön kameraya GİL yerleştirilmesi nispeten kolay gözükse de kornea dekompansasyonu, UGH sendromu ve kistoid makula ödemine neden olma eğilimi yüksektir (10,11). Bu nedenden dolayı sekonder GÍL yerleştirilen hiçbir hastada bu yöntem tercih edilmedi. İris fiksasyonlu GíL implantasyonu teknik olarak daha zor ve daha fazla zaman alsa da yapılan çalışmalara ameliyat sonrası sonuçların daha iyi olduğunu göstermiştir (12,13). Azda olsa en s1k görülen komplikasyonlar GİL dislokasyonu, endotel hücre kayb1, kistoid makula ödemi (KMÖ) ve UGH sendromudur (13). İris fiksasyonu yapılan hastalardan sadece bir hastada komplikasyon olarak UGH gelişti. Diğer hastalarda sonuçlar sulkusa yerleştirilen hastalar kadar iyiydi ve hiçbir hastada komplikasyon izlenmedi. Skleral fiksasyonlu GİL implantasyonunda, GİL skleraya fibrin yapıştırıcı ya da sütürle fikse edilebilir $(14,15)$. Ayrıca, ön kamera GİL'lerden teknik olarak yerleştirilmeleri daha zor olmakla birlikte, arka kamara yerleşimi nedeniyle uzun vadeli komplikasyon oranlarının daha düşük olduğu düşünülmektedir
$(16,17) . \mathrm{Bu}$ yöntemin görülen en sık komplikasyonları KMÖ, glokom, sütür kopması ya da erozyonu şeklindedir $(18,19)$. Çalışmamızda skleral fiksasyon yapılan bir hastada sütür kompası nedeniyle hastaya GIL repozisyonu yapıldı. Diğer iki yöntemle karşılaştırıldığında hastalarda astigmatizma düzeyinin biraz daha yüksek olduğu görüldü. Bunun da sütürasyon sirasında GÍL'in biraz daha tilt pozisyonunda olması ya da sütürün gergin olması ile ilgili olabileceği düşünüldü.

Katarakt cerrahisi sonrası en azından uzak görmede gözlüksüz bir hayat, hasta memnuniyetinin önemli parametrelerinden biridir. Komplikasyonsuz katarakt cerrahilerinde, her ne kadar GILL yerleştirilmesi standart olarak uygulansa da, kapsül bütünlüğünün bozulduğu durumlarda alternatif yöntemleri düşünmek bu açıdan önem arz etmektedir. Yapılan yöntemler içerisinde sulkus yerleşimli GİL implantasyonu, hastanın yeterli sulkus desteği olması durumunda hem daha kolay hem de cerrahi sonuçlar açısından daha başarılıdır. Kapsül desteğinin yetersiz olduğu durumlarda, iris fiksasyon ile skleral fiksasyonlu GIL implantasyonu tercih edilebilir. Cerrahın tecrübesine göre hastaya aynı seansta yâda ikinci bir seansta işlem yapılabilir. Bunun mümkün olmadığı durumlarda, ön kameraya GİL yerleştirilmesi yerine alternatif bu yöntemlerin yapılabileceği merkezlere hastaların refere edilmesi, olabilecek komplikasyon riskini azaltmak ve tekrarlayan cerrahileri önlemek açısından önemlidir.

\section{KAYNAKLAR}

1. Elfiky M, Saad H, Elseht R, Selima A. Role of ultrasound biomicroscopy in the planning for secondary implantation of intraocular lens in aphakia. Int Ophthalmol. 2016;36(3):391-400.

2. Chan TCY, Lok JKH, Jhanji V, Wong VWY. Intraocular lens explantation in Chinese patients: different patters and different responses. Int Ophthalmol. 2015;35(5):679-84. 
3. Jones JJ, Jones YJ, Jin GJC. Indications and outcomes of intraocular lens exchange during a recent 5-year period. Am J Ophthalmol. 2014;157(1):154-62.

4. Jin GJC, Crandall AS, and Jones JJ. Intraocular lens exchange due to incorrect lens power. Ophthalmology. 2007;114(3):417-24.

5. Fernández-Buenga R, Alió JL, Pinilla-Cortés L, Barraquer RI. Perioperative complications and clinical outcomes of intraocular lens exchange in patients with opacified lenses. Graefes Arch Clin Exp Ophthalmol. 2013;251(9):2141-6.

6. Schulze S, Bertelmann T, Sekundo W. Implantation von Intraokularlinsen in den Sulcus ciliaris. Ophthalmology. 2014;111(4):305-9.

7. Chang SHL, Wu WC, Wu SC. Late-onset secondary pigmentary glaucoma following foldable intraocular lenses implantation in the ciliary sulcus: a long-term follow-up study. BMC Ophthalmol. 2013;13(1):22.

8. Friedman NJ, Khater TT, Koch DD. Secondary intraocular lens implantation. In (eds): Philadelphia, PA: Lippincott, Williams, \& Wilkins, 2006.

9. Sorenson R, Scott IU, Tucker SH, Chinchilli VM, Papachristou GC. Practice patterns of cataract surgeons at academic medical centers for the management of inadequate capsule support for intracapsular or sulcus intraocular lens placement during cataract surgery. J Cataract Refract Surg. 2016;42(2):239-45.

10. Drolsum L. Long-term follow-up of secondary flexible, open-loop, anterior chamber intraocular lenses. J Cataract Refract Surg. 2003;29(3):498503.

11. Lyle WA, Jin JC. Secondary intraocular lens implantation: anterior chamber vs posterior chamber lenses. Ophthalmic Surg. 1993;24(6):37581.

12. Chen Y, Liu Q, Xue C, Huang Z, Chen Y. Threeyear follow-up of secondary anterior iris fixation of an aphakic intraocular lens to correct aphakia. J Cataract Refract Surg. 2012;38(9):1595-601.

13. Rusu I, Chen Z, Zizva J, Myung JS, Wald KJ. Incidence of cystoid macular edema with irisfixated posterior chamber intraocular lenses in patients presenting with lens dislocation. Int Ophthalmol. 2014;34(5):1153-8.

14. Rho S, Song WK, Sung Y, Kwon HJ, Lew H. Scleral fixation technique using a hydrophobic foldable intraocular lens with ring-shaped connecting bridges. J Cataract Refract Surg. 2015;41(2):262-7.

15. Ganekal S, Venkataratnam S, Dorairaj S, Jhanji V. Comparative evaluation of suture-assisted and fibrin glue-assisted scleral fixated intraocular lens implantation. J Refract Surg. 2012;28(4):249-52.

16. Kjeka O, Bohnstedt J, Meberg K, Seland JH. Implantation of scleral-fixated posterior chamber intraocular lenses in adults. Acta Ophthalmol. 2008;86(5):537-42.

17. Evereklioglu C, Er H, Bekir NA, Borazan M, Zorlu F. Comparison of secondary implantation of flexible open-loop anterior chamber and scleralfixated posterior chamber intraocular lenses. J Cataract Refract Surg. 2003;29(2):301-8.

18. Mimura T, Amano S, Sugiura T, Funatsu H, Yamagami S, Oshika T et al. 10-year follow-up study of secondary transscleral ciliary sulcus fixated posterior chamber intraocular lenses. Am J Ophthalmol. 2003;136(5):931-3.

19. Price MO, Price FW, Werner L, Berlie C, Mamalis $\mathrm{N}$. Late dislocation of scleral-sutured posterior chamber intraocular lenses. J Cataract Refract Surg. 2005;31(7):1320-6. 